\title{
ATTITUDE RESTITUTION FOR MULTISPECTRAL EARTH OBSERVATION SATELLITE USING SPACE TRIANGULATION TECHNIQUE
}

\author{
N. Bernardini ${ }^{1}$, R. Binet ${ }^{2}$, B. Vidal ${ }^{2}$ \\ ${ }^{1}$ University of Surrey, GU2 7XH Stage Hill, Guildford, United Kingdom - n.bernardini@ surrey.ac.uk \\ ${ }^{2}$ CNES, 18 Avenue Edouard Belin, Toulouse, France - renaud.binet@cnes.fr - bruno.vidal@cnes.fr
}

\section{Commission TC-I}

KEY WORDS: Attitude correction, Image Geometry, Space Triangulation, Multispectral Observation.

\begin{abstract}
:
Earth observation satellites are equipped with navigation components (star trackers, gyroscopes) which estimates the attitude of the satellite, corresponding to its orientation in the orbital reference frame. This equipment is essential for the localisation of the images which is done a posteriori and for the registration of the different images for a pushbroom sensor. This concerns both the registration of the spectral bands and the registration of multi-temporal series. However, the required accuracy is not always compatible with the performance of the attitude restitution. Moreover, some specific applications need more stringent geometric control even if requirements are met. With a multi-spectral pushbroom instrument, the same object on ground is seen as many times as number of spectral bands at different time instants: the attitude profile of the platform can be completely reconstructed from the results of the mapping of the different spectral bands. An attitude reconstruction method is proposed based on space triangulation inversion technique. The framework of this study is low-frequencies noise perturbations applied to Ven $\mu$ s satellite. Cubic splines are used for the attitude error profile, with 60 free parameters. Provided the physical model is representative, we show on two test cases that the convergence is very good. The band registration quality is used as a proxy to assess the performances. Residuals errors are less than 0.05 pixel for all tested band couples.
\end{abstract}

\section{INTRODUCTION}

Different types of sensors can be adopted to perform Earth observation. One of the most used is the push-broom. Because a pushbroom instrument uses satellite motion to create the image columns, it is necessary to have a precise attitude and orbit control system (AOCS). This system is widely used on nongeostationary satellites such as Pleiades, Sentinel-2, Ven $\mu$ s, and many more.

Errors on the attitude knowledge affect the inner image raw geometry, degrading the capability to register two images or two spectral bands in a common reference grid. For this reason, it can be necessary to estimate the satellite attitude error profile in order to regain the desired image registration performance. This paper aims to explain a methodology to estimate the attitude error profile of one acquisition using only 3 spectral bands, based only on image processing.

This paper focuses on the Earth observation satellite called Ven $\mu$ s (Vegetation and Environment on a New $\mu$ Satellite) which was launched in 2017. This satellite has been developed by the French Space Agency in collaboration with Israel Space Agency. The goal of Ven $\mu$ s mission is to study the vegetation of 123 selected sites worldwide with a repeat cycle of 2 days and with a constant viewing angle for each site. The main goal of the mission is to map the vegetation and to study its evolution in time. To perform these studies, the multi-band registration must reach an accuracy of 0.2 pixels rms and multi-temporal registration requires 0.3 pixel rms. The satellite experiences attitude restitution errors which hampers the geometric image quality. A ground correction method has been setup by CNES at the end of in-orbit calibration campaign (Binet, 2018) and this study deals with a drastic enhancement of the geometric correction, opening new fields of applications.

A few studies can be found in the literature regarding attitude estimation with a pushbroom instrument by image processing (Perrier, 2014) (Zhen, 2019) (Delvit, 2012) (de Lussy 2008)
(Roques, 2001): they usually use different detectors of the same flying instrument which have a fixed delay between the acquisition of a particular ground point. Usually these detectors share the same focal plane (for instance Pleiades PAN/XS bands). The basic idea is that the different detectors experiencing the same attitude error at different image lines, analysis of the landscape misregistration on these images yields the differential attitude over the imaging delay. These studies deal with attitude errors that are stationary during the time delay between spectral bands. Moreover in most publications (Zhen, 2019) (Delvit, 2012) (de Lussy 2008) (Roques, 2001), the attitude perturbations are quasi-harmonic jitter occurring at a frequency higher than the AOCS sampling frequency (typical value $10 \mathrm{~Hz}$ ). In the case of this study, the attitude error comes from the noise of the AOCS equipment which is low frequency. A particularity of Ven $\mu$ s is also the $2.7 \mathrm{~s}$ delay which is necessary to cover all the spectral bands for a given ground point, compared to a hundred milliseconds in the case of Pleiades. With such delay we cannot state that the attitude errors are stationary. This is why we propose another method to cope with these attitude errors.

The main technique that is discussed is space triangulation, which is commonly used for space pushbroom sensors for bundle adjustments and/or geolocation refinement. Some works have already been done on the topic of Ven $\mu$ s attitude correction using space triangulation technique. Polynomial functions were used to estimate the attitude errors thanks to ground control points (GCPs) taken automatically on a reference image (Binet, 2018). This correction is operated systematically at the Ven $\mu$ s ground segment. However, the implemented method happens to be not sufficient for all cases; in fact $20 \%$ of Ven $\mu$ s images have not been fixed. This issue is mainly created by the intrinsic limitations of the fitting function (polynomial) with respect to the real perturbation. This paper aims to show the possibility to apply cubic splines to estimate the attitude error profile. It is demonstrated that the algorithm converges to a solution that is a great improvement over the one obtained using the previous method. 


\section{VEN $\mu$ S SATELLITE}

Launched in 2017, dedicated to vegetation and land monitoring, VEN $\mu \mathrm{S}$ scientific mission is a unique combination of specific features: high spatial resolution $(5 \mathrm{~m})$, high revisit (2-day orbital cycle), and high spectral resolution (12 spectral bands from 420 to $910 \mathrm{~nm})($ Dedieu, 2006). 123 sites on Earth, representative of different ecosystems and climatic zones, are currently acquired. Freely available on THEIA land data portal (https://www.theialand.fr/en/product/venus), VEN $\mu \mathrm{S}$ data provides opportunities to validate image processing algorithms and study the land surface evolution for a wide range of surface types (ice, vegetation, soils...).

\subsection{Venus Focal Plane Geometry}

The optical payload and its design has been reviewed in details in (Topaz, 2006). As shown in Figure 1 the detector module (or focal plane) is equipped with 4 tri-detectors. Each tri-detector includes 3 different detector arrays with their own spectral band making a total of 12 bands. Every detector array has 5200 pixels. The spectral bands distribution is shown in Figure 3.

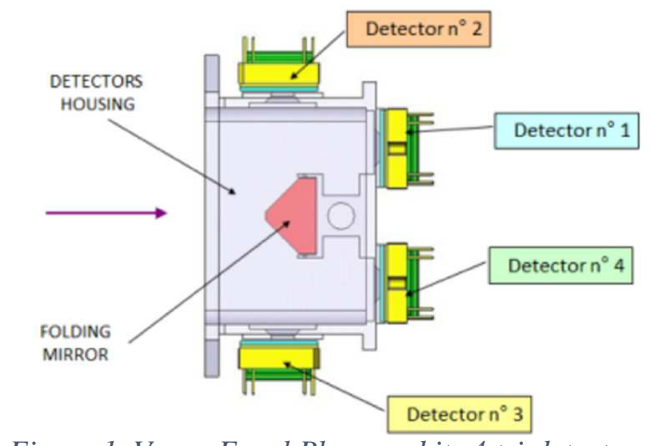

As shown in Figure 2, the different spectral bands see the same ground point at different times. Since the satellite has moved between two band acquisitions, it yields a stereoscopic parallax: the terrain height is then needed to finely register the band images. The maximum baseline over height $(\mathrm{B} / \mathrm{H}$ ratio) is met with B05/B06 couple with a value of 0.025 . As one can see in Figure 3, B05 and B06 are two special detectors sharing the same spectral band for low baseline stereoscopic capability purpose.

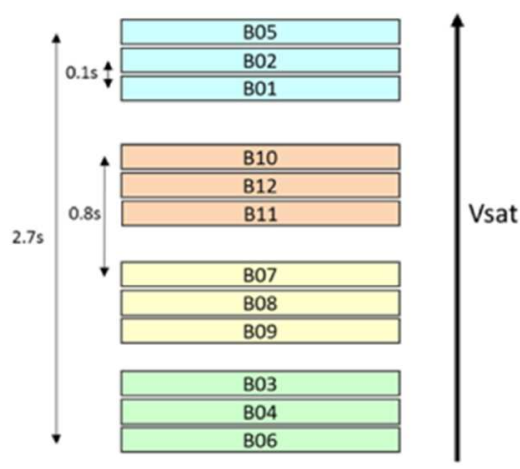

Figure 2. Venus Bands Distribution Projected on Ground and Time Intervals

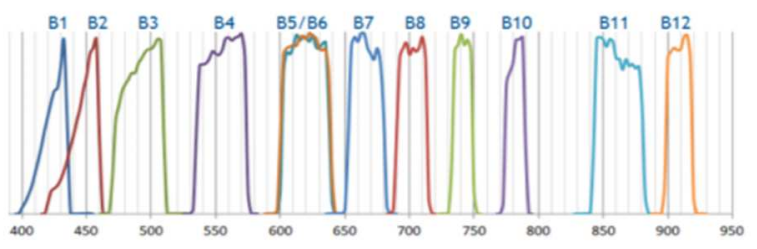

Figure 3. Venus Spectral Bands. Notice that B5 and B6 share the same spectral band.

\subsection{Venus Attitude Error and its Consequences on Image Quality}

Ven $\mu$ s mission is affected by a strong low frequency attitude noise as shown in Figure 4 (beginning of life). This noise hampers the orientation knowledge of the instrument boresight during time. Since it is a pushbroom system with asynchronous band acquisitions, this noise has a great impact on the on-ground band registration capability. Because this noise changes from one acquisition to another, it has also an impact on the multi-temporal registration quality. In-orbit calibration of the pixel's line of sight was also a challenge (Binet, 2018).

The most stringent requirement is the multi-band registration quality. We have to correct these attitude errors such that the band misregistration error is below 0.2 pixels rms for all couples of bands.

Since this noise is band-limited $(5 \mathrm{~Hz})$, the multi-band registration requirement is met for short delays band couples, typically 2 bands inside a tri-detector. But this requirement is not met for band couples involving different tri-detectors, having larger delays (delays are sketched on Figure 2). The worst band registration performance happens with B05-B06 couple, which has the maximum $2.7 \mathrm{~s}$ delay.

As a collateral damage, DEM created with B5/B6 detectors are strongly affected by a typical height noise of $100 \mathrm{~m}$, which is unusable for most applications.

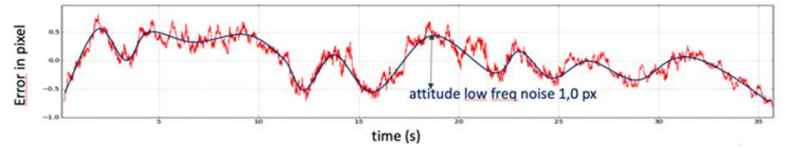

Figure 4. Column misregistration of B05 and B06 on a very long scene (35s) at beginning of life, with navigation data correction. Red profile is the measured misregistration, showing the $5 \mathrm{~Hz}$ attitude restitution noise. The black profile is a smoothed profile showing the low frequency attitude noise. Peak to peak noise can reach 1 pixel.

\section{ATTITUDE CORRECTION}

\subsection{Actual Geometric Corrections of Ven $\mu$ s Products}

Geometric corrections have been implemented on Ven $\mu$ s ground segment in order to reach a good registration and geolocation of distributed L1 ortho-products, based on CNES experience on former projects.

The attitude noise was expected to be no more complex than a temporal drift for each of the 3 components (roll, pitch, yaw). In a space observation context, small orbital restitution biases (typically less than $10 \mathrm{~m}$ ) can be assimilated to attitude biases. Instrument pixel line of sight (LOS) are calibrated in an instrument frame and are considered static over a year, updated 
if needed. LOS calibration errors are typically less than 0.05 pixel.

Space triangulation technique (also known as bundle adjustment), associated with a physical rigorous model, is generally well suited for attitude correction. GCPs are automatically extracted from a reference image, being a chosen cloud-free Venus image. GCPs height are extracted from Planet Observer DTM, which is slightly better than SRTM3s. DTM height errors do not hamper co-registration of spectral bands because stereoscopic baseline $(\mathrm{B} / \mathrm{H})$ is very low. Multi- temporal registration is also not hampered by GCPs height errors because Ven $\mu$ s acquires with the same orbit and same viewing angle each scientific site.

For drift and biases attitude correction, polynomial models of degree 1 for each axis are perfectly suited, provided sufficient GCPs are found. On cloud free images, we experience thousands of GCPs per image, which is much more than we need to estimate the 6 attitude parameters.

Dealing with actual attitude errors, the best economic solution was to increase the order of the polynomials up to degree 5 . (Binet, 2018) describes in details the whole process and results.

After all, this kind of attitude correction do benefit to the overall image geometric quality: Ven $\mu$ s images fullfill the registration requirements for more than $80 \%$ of all distributed images. This paper addresses the problem of a better correction in order to be able to use B05/B06 stereoscopic capabilities, and to open new fields of applications having strong geometric requirements.

\subsection{The Geometric Model}

There are different models that can be used for image geometry. These models can be categorised in two groups: high accuracy image geometry models and "approximated" image geometry model. In this paper we focused our attention on the rigorous physical model. A rigorous physical model allows to have a nearly-optimal adjustment and to reach accuracies of fractions of pixels which is the amplitude of errors we are interested in.

The physical model is composed by the following parameters:

- $\quad$ time of the pixel acquisition

- satellite position function of time

- $\quad$ satellite attitude function of time

- pixel line of sight in the instrument frame (inner orientation)

We define function $\mathrm{F}$ as the direct localisation model:

$$
(\lambda, \phi)=F\left(l, c, h, P_{1}^{0}, \ldots, P_{k}^{0}, \ldots, P_{n}^{0}\right)
$$

Where $(\lambda, \phi)$ refers to longitude and latitude, 1 refers to the timevarying sampling number (line index), c refers to the pixel position on the pushbroom sensor (column index), and $P_{k}^{0}$ terms represent the acquisition parameters, including exterior orientation function of time and a fixed instrument inner orientation. In the following, $P_{k}^{0}$ refers to measured parameters coming from navigation data (exterior orientation) and in-orbit calibration campaign (inner orientation).
We also define the inverse localisation function $\mathrm{G}$ :

$$
(l, c)=G\left(\lambda, \phi, h, P_{1}^{0}, \ldots, P_{k}^{0}, \ldots, P_{n}^{0}\right)
$$

To perform both direct and inverse localisation, collinearity equations are used. No atmospheric refraction correction nor light travel time correction have been considered in this study because at the scale of a Venus image, these phenomena induce a global location bias which can be assimilated to a constant orientation error, hence a global attitude error.

In order to refine the geometric model, we introduce additional error parameters in the model that will be refined by space triangulation. $P_{k}^{0}$ parameters then become $P_{k}^{0}+d P_{k}$ with $P_{k}^{0}$ the initial parameters and $d P_{k}$ the error parameters we want to refine.

In space triangulation technique, error terms are refined thanks to measurements on specific image homologous points that are tie points and/or GCPs.

\subsection{Measurements: GCPs and Tie Points}

\subsubsection{Homologuous Points Processing}

Homologous points are created by sub-pixel windowedcorrelation between a raw reference image and another raw slave image (either two spectral bands or a spectral band with a reference image). The homologous points are computed every 20 pixels step in row and column. The reference image is resampled into slave image raw geometry using the initial geometric model and a DTM. Care is given to resampling quality in order to avoid aliasing artefacts. The valid homologous points of the reference image are reprojected back into its native raw geometry in order to create tie points measurements. For GCPs, we finally compute the $3 \mathrm{D}$ geolocation of the reference tie points by application of direct location model on the DTM.

\subsubsection{GCPs Extraction}

The GCPs are extracted between one band of the Venus image and a reference image whose geolocation is supposed perfect. The reference image is a Sentinel-2 GRI extract (Dechoz, 2015), having $10 \mathrm{~m}$ ground sampling distance, being twice the Venus pixel size. For fine attitude restitution purposes, we do not choose a previous Ven $\mu$ s image because we do not want to mix reference image errors with the one of the processed image. Considering Ven $\mu$ s swath $(26 \mathrm{~km})$ with the one of a single Sentinel-2 detector array $(25 \mathrm{~km})$, we have enough overlap between the 2 images in order to extract a high number of GCPs over the full acquisition time of Ven $\mu$ s image. Taking a single detector array of raw Sentinel-2 acquisition is good for reducing inter-detector discrepancies. However, this reference image is also potentially affected by Sentinel-2 attitude noise. The image matching accuracy is also hampered by the landscape changes and the resolution discrepancy. In addition, it is not always possible to identify GCPs; for example, due to changes in the vegetation during time, the reference image does not match the new ones, or in case of homogeneous surfaces, such as the sea or clouds, no GCPs can be defined. We compute two sets of GCPs, respectively with $\mathrm{B} 05$ and $\mathrm{B} 06$ bands, because the temporal coverage of B05 and B06 encompass all the spectral bands acquisition time.

Because of all these limitations, GCPs are intended to constrain only the absolute localisation and not the attitude profile. As such we set a low confidence to the GCPs (compared to the tie points). 


\subsubsection{Tie Points between Spectral Bands}

Tie points can be used to adjust several images simultaneously and find their relative positioning with respect to themselves. For our problem, attitude variations could be solved with tie points only. GCPs are only needed for absolute geolocation.

Contrary to GCPs measurements, tie points computed between spectral bands do not suffer from temporal decorrelation, and do not introduce additional external errors. As such, their matching accuracy is much higher and the confidence of these measurements is also much better than the one of GCPs.

However, because of landscape spectral variations, accurate tie points can only be retrieved in appropriate spectral bands couples. The best band couple regarding image correlation and attitude inversion is B05-B06 thanks to the same spectral sensitivity and to the highest time gap of $2.7 \mathrm{~s}$. B05-B07 is also a very good choice thanks to very close spectral bands and an intermediate time gap.

Repeating the homologous retrieval process with different band couples and using the same points of interest, we can create $\mathrm{N}$ uplets tie points, which is better for the space triangulation because we need to solve only one ground position for each $\mathrm{N}$ uplet instead of one per couple. Using 3 bands instead of 2 also solves the height-attitude ambiguity discussed in \$3.4.4.

In this study we computed B05-B06-B07 triplets tie points on the whole field of the image whenever possible (water and clouds forbidden). The B09/B10 couple has also been used for the attitude correction assessment.

\subsection{Attitude Restitution}

\subsubsection{Space Triangulation}

Space triangulation, commonly used for bundle adjustment (Triggs, 2010), makes use of the ground control points and tie points in order to refine acquisition parameters. The GCPs yield the absolute image coordinates and therefore they ensure the absolute orientation for the segment while the tie points allow to find the relative placement between two or more images. The method allows to estimate simultaneously the geometric unknowns, the terrain coordinates and the image coordinates of the points (Figure 5).

To find the solution it is necessary to solve a set of 3 types of equations. The first set of equations are the image equations, or observation equations which are obtained for each measurement $\left(l_{\text {meas }}, c_{\text {meas }}\right)$ of point $\mathrm{M}$ in image I; For a ground control point the equation is the following:

$$
\begin{gathered}
d I=I_{\text {meas }}-I_{\text {est }} \\
=I_{\text {meas }}-G_{I}\left(X_{G C P}, Y_{G C P}, Z_{G C P}, P_{1}, \ldots, P_{k}, \ldots, P_{n},\right. \\
\left.d P_{1}, \ldots, d P_{k}, \ldots, d P_{n}\right)
\end{gathered}
$$

Where $G_{I}$ is the inverse geometric model for image I, Imeas (resp. $\mathrm{I}_{\text {est }}$ ) is the image measured (resp. estimated) position, and $d P_{k}$ are geometric model error terms.

While for a $\mathrm{N}$-upplet tie point we have $\mathrm{N}$ similar image equations

$$
\begin{aligned}
& d I_{1}=I_{\text {meas } 1}-G_{I_{-} 1}\left(X_{\text {est }}, Y_{\text {est }}, Z_{\text {est }}, P_{1}, \ldots, P_{k}, \ldots, P_{n},\right. \\
& \left.d P_{1}, \ldots, d P_{k}, \ldots, d P_{n}\right)
\end{aligned}
$$

$$
\begin{gathered}
d I_{j}=I_{\text {meas } j}-G_{I_{-} j}\left(X_{\text {est }}, Y_{\text {est }}, Z_{\text {est }}, P_{1}, \ldots, P_{k}, \ldots, P_{n},\right. \\
\left.d P_{1}, \ldots, d P_{k}, \ldots, d P_{n}\right) \\
d I_{N}=I_{\text {meas } N}-G_{I_{-} N}\left(X_{\text {est }}, Y_{\text {est }}, Z_{\text {est }}, P_{1}, \ldots, P_{k}, \ldots, P_{n},\right. \\
\left.d P_{1}, \ldots, d P_{k}, \ldots, d P_{n}\right)
\end{gathered}
$$

Where $\left(X_{\text {est }}, Y_{\text {est }}, Z_{\text {est }}\right)$ are tie points ground position unknowns.

The second type of equations are stress equations which enable to estimate new parameters near an initial guess. For a GCP, terrain stress equations enable to estimate the new point ground position near the GCP measurement. There are 3 times as many equations as number of GCPs.

$$
\begin{gathered}
d X=X_{\text {meas }}-X_{\text {est }} \\
d Y=Y_{\text {meas }}-Y_{\text {est }} \\
d Z=Z_{\text {meas }}-Z_{\text {est }}
\end{gathered}
$$

For a tie point, we can also add an initial altitude $Z_{\text {meas }}$ : it gives as many equations as number of tie points. This is an option we will discuss later.

Similarly we have stress equations for the parameters $d P_{k}$ which enable to limit the amplitude of error terms. Implicitly, we look for a solution near the initial one.

The two types equations (image equations and stress equations) have to be weighted by the uncertainties $\sigma_{X Y}$ for the planimetric measurement and $\sigma_{z}$ for the altimetric measurement. Similarly the image measurements are weighted by the position uncertainty $\sigma_{I}$ of $\mathbf{M}$ in the image, which is an estimation of the matching noise. The third type of equations are the stiffness equations, also called stress equations, for $d P_{k}$ unknowns weighted for the accuracy $\sigma_{p}^{k}$.

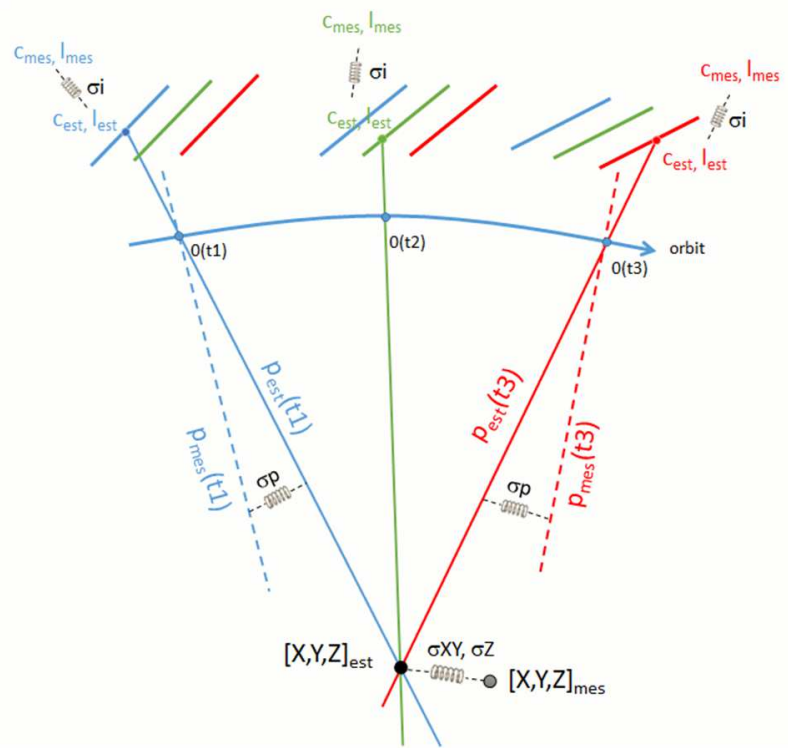

Figure 5 Principle of space triangulation applied to a single multiband pushbroom acquisition. Given measured image tie points and GCPs acquired in different bands at different times and attitude parameters $p_{m e s}(t)$, we estimate a new set of image ground coordinates and attitude parameters pest $(t)$ that are consistent with collinearity equation and measurements error $\sigma p, \sigma i, \sigma X Y, \sigma Z$. 


\subsubsection{Least Square Solving}

We want to find a solution of $d P_{k}$, together with new image and ground positions that minimizes the residuals $d P_{k} / \sigma_{p}^{k}, d I /$ $\sigma_{I}, d X / \sigma_{X Y}, d Y / \sigma_{X Y}, d Z / \sigma_{Z}$ in a least square sense.

The system is non-linear and it is required to be linearized with respect to unknowns. For sake of clarity we simplify the notation and $p$ states for $d P_{k}$ parameters and $s$ states for terrain coordinates $\left(X_{\text {est }}, Y_{\text {est }}, Z_{\text {est }}\right)$. Image equation become:

$$
\begin{aligned}
d I= & I_{\text {meas }}-\frac{\partial G}{\partial p} \delta p-\frac{\partial G}{\partial s} \delta s-G\left(p_{0}, s_{0}\right) \\
& =-G_{\text {lin }}(\delta p, \delta s)+\left(I_{\text {meas }}-I_{0}\right)
\end{aligned}
$$

Where $I_{0}$ is the current guess for image coordinate. This equation can be represented in a matrix way as $A x-b=v$, where v are the residuals, and $\mathrm{x}$ are the unknowns $(\delta p, \delta s)$. We can use this linear solution to estimate iteratively the final estimation. Finally the minimisation problem becomes equal to the minimisation of $(A x-b)^{T}(A x-b)$ which is equal to solve the following normal equation:

$$
A^{T}(A x-b)=0
$$

Levenberg-Marquardt algorithm has been adopted as optimizer (Gavin, 2019).

The algorithm has been implemented in a new JAVA library, called GeoPix which has enabled us to perform this study.

\subsubsection{Cubic Splines Attitude Restitution}

To estimate the attitude error of the satellite it is necessary to first define the error function that describes the satellite attitude error profile. Previous work has been done using polynomial functions, one for each rotation axis (roll, pitch, yaw). However polynomials are usually limited due to the fact that they are not capable to properly fit the desired signal; even increasing the polynomial degree does not help improving its performance due to the comparison of Runge's phenomena. That is why we limited to a degree 5 the polynomial attitude correction at Venus production center.

In this paper we tested cubic splines error functions for each attitude axis. Indeed it is suited to the low frequency noise we want to cancel. These functions are parametrized by $\mathrm{N}$ samples regularly spaced in time, covering the acquisition time interval.

Given attitude error of every sample and adding constraints at the first and last samples, there is a unique set of cubic splines that describes the attitude error profile. As for the constraints at edge's samples, the second derivative is set to 0 . We add samples around the acquisition time interval so that effective error profile is not limited by these constraints.

In our experiments, the dates of the samples are fixed a priori given a sampling frequency. This frequency shall be high enough in order to fit properly the true attitude error profile. In our case 30 points per axis is a good compromise between computation time and correction performance. This number corresponds to a sampling of $\sim 5 \mathrm{~Hz}$, which is approximately twice the bandwidth of our error.

Following our definitions, the $d P_{k}$ parameters that we want to estimate are the attitude errors on the $\mathrm{N}$ samples.

\subsubsection{Separation of height and pitch angle}

Tie points height estimation and pitch attitude profile are strongly correlated for 2 bands. Indeed, for a pushbroom device, a pitch attitude drift modifies the time interval at which a ground point is seen in the 2 bands, hence the stereoscopic baseline.

(Zhen, 2019) suggested to add a third band in order to separate height and pitch attitude component. Similarly we use tie points involving 3 bands (triplets) in order to better constrain the problem.

\section{VEN $\mu S$ ATTITUDE CORRECTION}

\subsection{Ven $\mu$ s Attitude Pre-Filtering}

The Ven $\mu$ s attitude error is characterised by frequencies up to $5 \mathrm{~Hz}$ caused by the onboard attitude sensors. Hopefully the real attitude of the platform is much smoother, and Venus do not experience high frequency jitter. For this reason, we propose to perform a "radical" quaternion filtering by fitting each quaternion component by a third degree polynomial. This filtering degrades the geometric quality of the image in terms of error amplitude because obviously the filtered attitude is not accurate. But the resulting attitude error profile, which is the "difference" between real attitude and filtered attitude is smoother : the error amplitude is greater but the error profile bandwidth has been divided by a factor $\sim 2$. Therefore fewer free parameters are needed to solve the attitude error profile.

\subsection{Geometric Error Model}

The geometric error model parameters of Ven $\mu$ s images are setup thanks to our experience after the in-orbit calibration campaign.

The attitude error profile has been decomposed into the 3 rotations angles (pitch, roll, yaw) in the satellite reference frame. To simplify the problem, we have neglected the estimation of the yaw rotation as it is a second order error. To estimate the remaining rotations, we have used 30 cubic splines both for the pitch or the roll axis. Cubic splines profiles are entirely defined by 30 time samples regularly spaced in time. The refined parameters are the roll and pitch values of these samples. The number of splines is set high enough to fit the real attitude error after pre-filtering.

Until now we have only focused on attitude errors but the space triangulation algorithm can also invert other type of modelling parameters. Examples of other errors are pixel time tagging error, orbit position error, detector position error in the focal plane.

During this study, it has been required to introduce some additional errors to our model in order to improve algorithm convergence. Indeed, an error in our geometric model which is not assimilated to an attitude error would prevent the images from being perfectly registered, and the true attitude to be found.

Additional error models can be easily added as parameters in the optimisation. However, we have to be careful not to unconstraint the problem.

The error we focused our attention on is the tri-detector relative position error. This error is a relative angular error between 2 detectors belonging to 2 different tri-detectors. Detector relative positions in the focal plane are correlated with the attitude drift in pitch and roll axes. Consequently, the in-orbit calibration of 
relative tri-detectors position has been a challenge (Binet 2018). Ignoring these static errors would result in an inconsistent attitude estimation. Moreover, it is likely that no attitude profile can compensate these static errors in order to have no registration residual. So if the detectors boresight calibration is not perfect, attitude profile and boresight calibration should be estimated together. Since the variables are correlated, this can be done only if we introduce a new constraint.

In this study this constraint is given by the terrain height rough knowledge. Indeed, the angular separation of the detectors yield a stereoscopic sensitivity. Therefore there is a direct relationship between tie point height retrieval and detectors angular separation, which cannot be separated without giving an initial guess of tie point height. In the space triangulation solver, these new measurements add as many stress equations as the number of tie points. The quality of the DTM should match the stereoscopic sensitivity of the imaging system. In Ven $\mu$ s case, baseline over height ratio is no more than 0.025 , therefore Planet Observer DTM is accurate enough to separate these parameters. It is to be noted that introducing this additional error model yielded a great improvement of the experimental results. We think this is a clue for the true presence of small errors of tridetector positioning after the in-orbit calibration campaign.

One tri-detector is fixed and the other tri-detectors can translate along the two directions of the focal plane. These errors are equivalent to roll and pitch bulk rotations of all the lines of sight of the 3 detectors considered.

\subsection{Performance Assessment}

Once the attitude of the satellite has been estimated it is necessary to assess its accuracy with the multi-band registration criterion. A new set of tie points, different from the space triangulation, is measured following the same processing (see section 3.3). Residuals are computed using the refined geometric model. Tie points are set on a regular grid in raw geometry over the shared area of the band couple. If the satellite attitude is perfectly estimated, these residuals should be null. Four error terms are awaited: attitude residuals, which are constant linewise, line of sight residuals which are constant columnwise, residuals due to DTM errors which are in our case quite low thanks to the low stereoscopic sensitivity, and residuals due to matching noise. The two last residuals are expected to be zero-mean, so that if we sum the residuals along the lines we get a profile of the attitude residuals, while if we sum along the columns we get a profile of the line of sight residuals. Typical profiles are shown in Figure 6 for 4 particular band couples before refinement. Left profiles of Figure 6 shows the along-track mean residuals profiles respectively in line and column directions. We can see the typical residual profile we want to cancel. The attitude has been preprocessed in order to filter out the noise coming from the navigation sensors. Thanks to this pre-processing, residuals cutoff frequency drops from $5 \mathrm{~Hz}$ to $\sim 2 \mathrm{~Hz}$ (see section 4.1 ). On the right of Figure 6, across-track mean residuals profiles reveal constant biases between bands, which are due to focal plane biases and/or a mean attitude drift error over the observation time. It is impossible to separate the two sources of error on such residuals analysis.
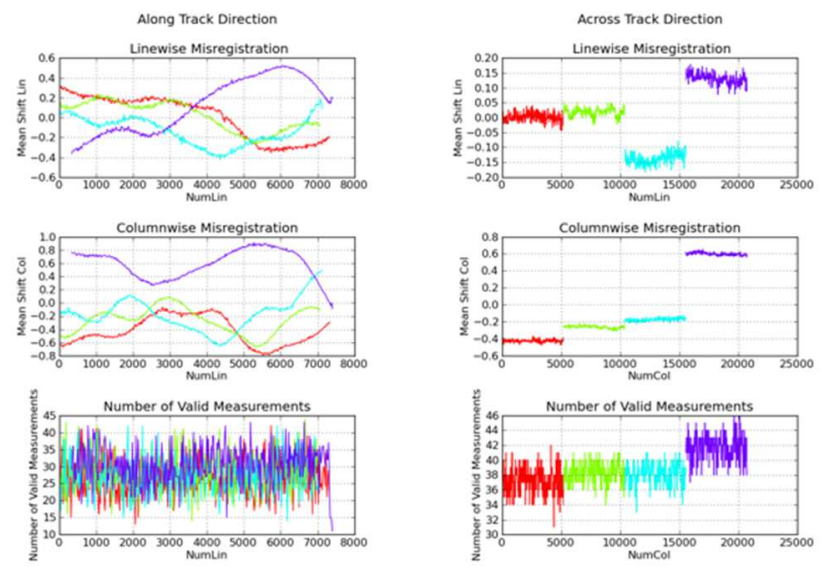

Figure 6 Example of misregistration profiles obtained with 4 spectral bands couples before attitude refinement and after quaternion filtering. The couples of bands used are B05-B07 (red), B06-B07 (blue), B09-B10 (green), B06-B05 (purple). Units are pixels.

For the residuals assessment, the spectral bands have been chosen in order to take into account all the tri-detectors. The couples of bands are: B05-B07 (red), B06-B07 (blue), B09-B10 (green), $\mathrm{B} 06-\mathrm{B} 05$ (purple). It is to be noted that the refinement is done only with B05, B06 and B07 measurements. B09-B10 measurements are used to control that the attitude profile is also applicable to the tri-detector $n^{\circ} 2$, eversince no measurements have constrained it to be good on this couple.

\section{RESULTS}

The results are shown on 2 Ven $\mu$ s test cases: a mountainous area whose nickname is FR-LQ1 and a seashore area named DUCK. The images are composed by $\sim 7000$ lines and 5200 columns, corresponding to $\sim 6 \mathrm{~s}$ of acquisition.

\subsection{Case 1: FRLQ1}

The chosen image corresponds to the site called FR-LQ1 which is located in the Massif Central, a highland region in the center of France. The scene does not contain clouds and is a good candidate to test the robustness of the proposed method over a mountainous area. Similarly to Figure 6, Figure 7 shows the registration performance after attitude filtering and before attitude refinement. The columnwise misregistration is higher than 1 pixel while linewise it goes from 0.4 to -0.3 pixels. The results without estimation do not match the requirements for a Ven $\mu$ s image, making the image unusable for scientific purposes as the error in pixel is larger than 0.2 pixel rms. It is clear that the additional correction is required to meet the desired performance.

In order to simulate the actual processing of Venus ground segment attitude correction, the first attitude estimation has been done with a polynomial of degree 5 as it is the fitting function which is used at Venus production center. The results shown in Figure 8 can be considered as a reference performance. As we can see on attitude residuals, high order terms cannot be fitted by such polynomial. Despite these oscillations, the registration requirements are met. However there is possibility for improvements. 

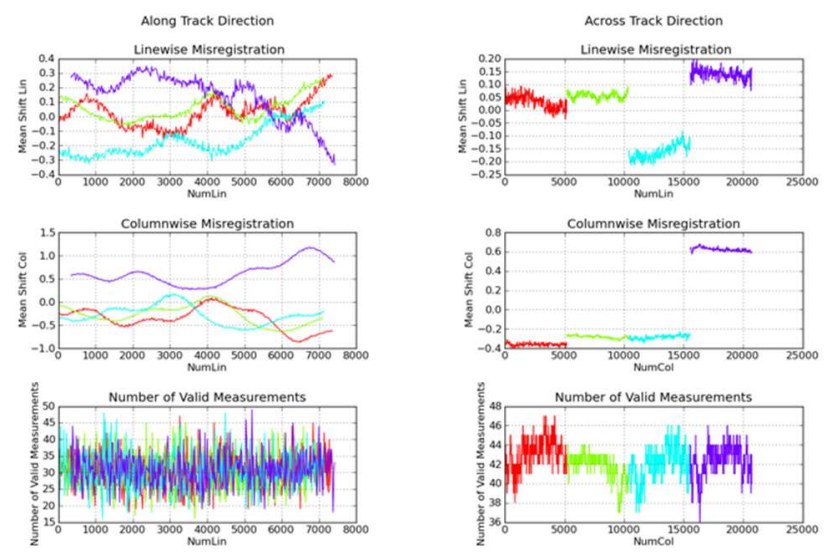

Figure 7 Misregistration profiles of FR-LQ1 image obtained with filtered attitude without attitude correction.
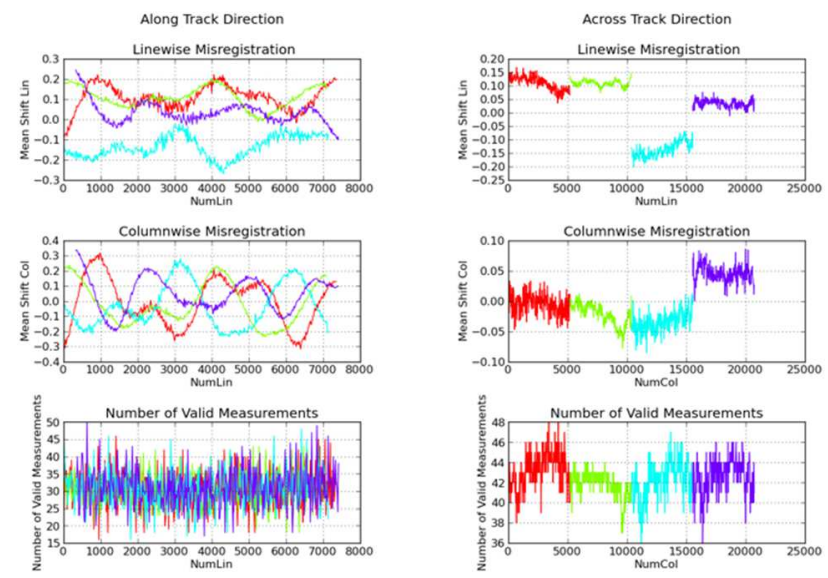

Figure 8 Misregistration profiles of FR-LQ1 image obtained with an attitude correction involving a 5 degrees polynomial function.
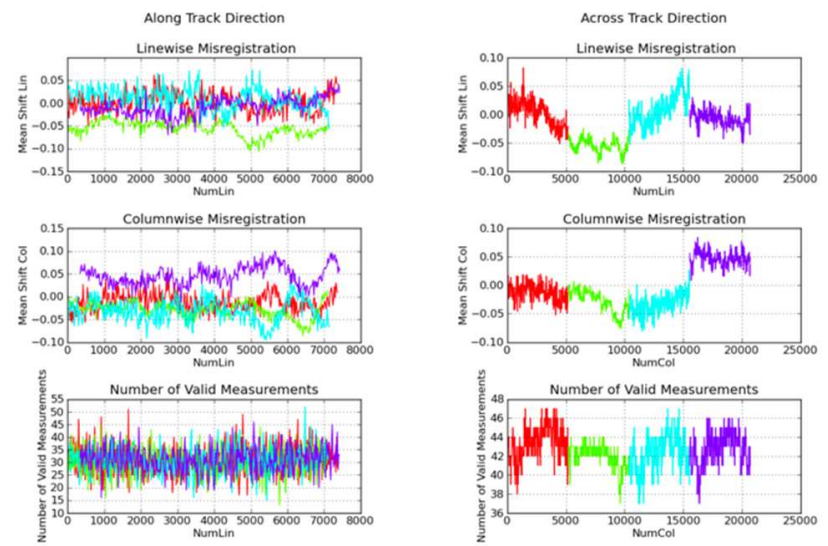

Figure 9 Misregistration of FR-LQ1 image obtained with an attitude correction involving 30 cubic splines and detectors position correction.

To improve the attitude restitution, we have adopted the new method using cubic splines. Results are shown on Figure 9. Comparing these results with the one from Figure 8 it is clear the spline allowed to greatly improve the results linewise and columnwise. In fact it is possible to say that the correction applied on the roll axes is almost perfect as the order of the error reaches
0.05 pixel which is 5 times less than the one requested. In both cases, spline and polynomials, it is possible to identify constant errors in the line of sight residuals that have not been corrected by our refinement. Maybe other sources of geometric noises are still present that avoid us to find a perfect physical solution.

\subsection{Case 2: DUCK}

The second site selected is named DUCK. The site is placed in South Carolina, USA. This nearshore site has been chosen to study the bathymetry thanks to local waves velocity estimation (Bergsma, 2019). This bathymetry inversion technique requires a very high confidence in the geometric quality, better than the Ven $\mu$ s requirements. However, the presence of water hampers the attitude refinement because it is not possible to measure GCPs or tie points above water. The resulting tie points repartition is sketched in Figure 12. We expect that the lack of tie points and the sparse repartition decreases the performance of the algorithm.

As in the previous case the attitude has been estimated using cubic splines. Figure 11 shows the residuals of the estimation done with 30 splines for the pitch and roll angles and including the detectors' position estimation. The resulting correction is far better than the one without correction shown in Figure 10. It is also clear that there is a series of lines between lines 7000 and 8000 for which there is no valid tie point because of the water cover. Nevertheless, it did not hamper the attitude estimation because the 3 band acquisitions being delayed, three homologous points are acquired at different dates separated by at least $1 \mathrm{sec}$, and there is no time interval in which we would have a loss of tie point. Such ill situation would occur with other imaging sensors having small time gaps between spectral bands.

Figure 11 shows the results of the estimation done with 30 splines for the pitch and roll angles and including the tri-detectors' position estimation. The obtained correction is able to give far better results compared with the results of Figure 10 without correction. The high residuals obtained on the couples B9-B10 and B6-B7 are due to the wrong tie points located on shoaling waves : it biases the performance estimation so these couples are not representative of the real registration performance. The two other band couples show very good registration results with zero mean errors in the line of sight profile and 0.05 pixel maximum error in the attitude residual profile. This performance is equal to the one of FRLQ1 test case. It tends to show that the limitation of the method on these two cases is not the number of tie points but rather the optimisation convergence (local minima) or the lack of representativeness of the geometric model.

\section{CONCLUSION}

This study has proved the ability to improve the band registration of Ven $\mu$ s imaging system. The proposed method outperforms the previous algorithm allowing to decrease the error up to 0.05 pixels. The convergence of the space triangulation optimization is good thanks to accurate tie points and a good modelling of the geometric model, yielding a well-constrained inverse problem. In our case cubic splines functions are well suited for the modelling of the attitude errors despite the consequent number of parameters to inverse. Particular attention has been put in the understanding of the conditioning of this inverse problem while adding so many degrees of freedom. 

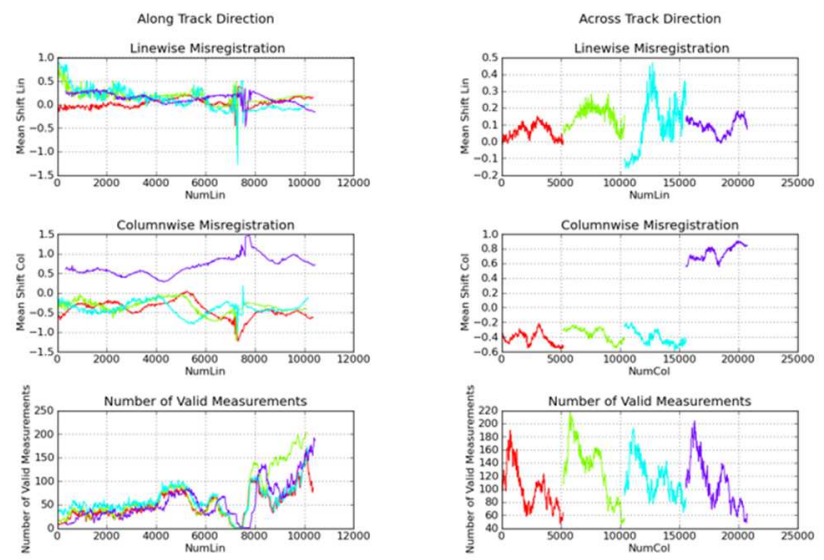

Figure 10 Misregistration profiles of DUCK image obtained with filtered attitude without attitude correction.
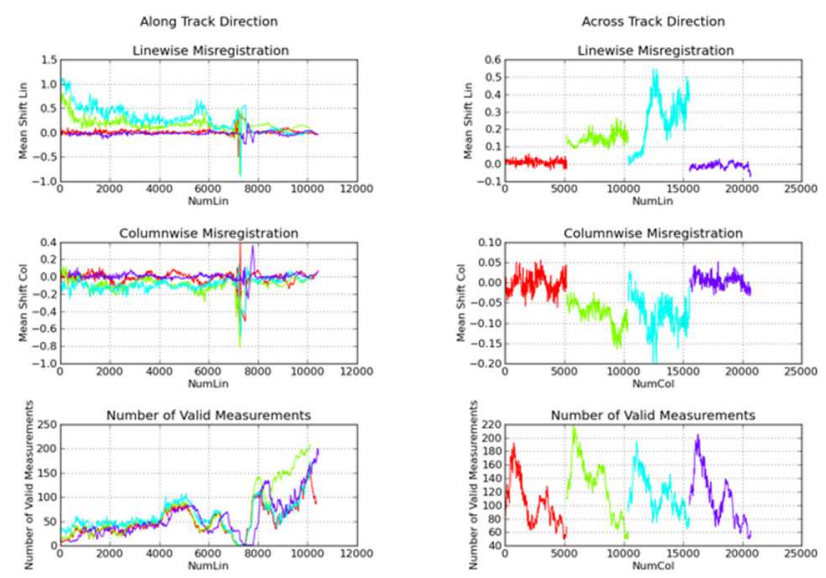

Figure 11 Misregistration of DUCK image obtained with an attitude correction involving 30 cubic splines and detectors position correction.
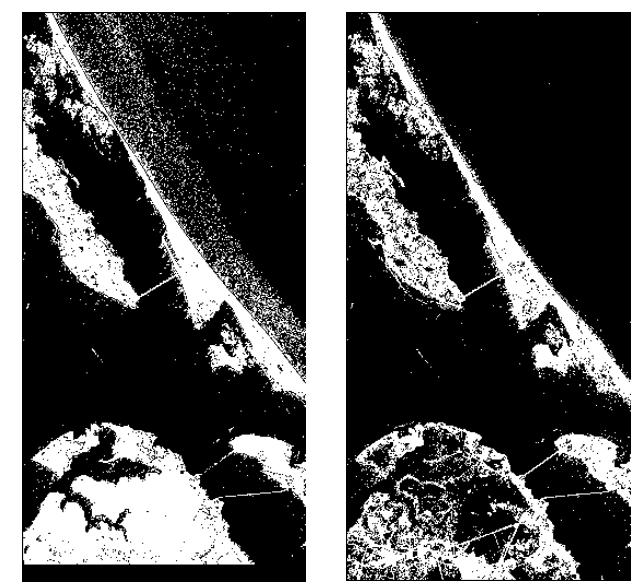

Figure 12 Validation Measurements on seashore site DUCK. Left: tie points repartition for B09-B10 couple. Right : tie points repartition for B05-B06 couple
The new library GeoPix allowed us a great flexibility (interpolation methods, errors parameters, terrain constraints) and we believe it can be easily adapted to other kinds of studies. It enabled to study the effects of parameters that until now had not been considered to be possible sources of errors like detector's angular separation. We believe we could go further ahead to enhance band registration since residuals are still measurable. For instance dating errors have not been studied yet.

The attitude correction of Ven $\mu$ s images yields a geometric accuracy which is far beyond the requirements. As such it enables new applications such as DEM estimation thanks to its native stereoscopic capability (Rolland, 2019), and bathymetry estimation thanks to appropriate time lags between the spectral bands (Bergsma, 2019). Other applications requiring subpixel geometric accuracy could benefit from this technique such as ground displacements measurements, or elevation changes measurements.

We believe that this kind of attitude correction could apply to other multi-sensors pushbroom instruments, even with a different design than the Ven $\mu$ s one. The large delay between Ven $\mu$ s sensors is adapted to low frequency attitude perturbation correction. Smaller sensor delays could solve high frequency oscillating perturbations and we are willing to test the correction on other platforms. The other perspective is to use space triangulation in order to calibrate accurately the pixel lines of sight thanks to a joint estimation of complex attitude correction and complex focal plane errors in a multi-acquisitions scheme.

\section{REFERENCES}

Bergsma, Erwin WJ, Rafael Almar, and Philippe Maisongrande. "Radon-Augmented Sentinel-2 Satellite Imagery to Derive Wave-Patterns and Regional Bathymetry." IGARSS Remote Sensing 11.16 (2019): 1918.

R. Binet, F. de Lussy, F. Languille, A. Rolland, P. Gamet, J-L. Raynaud, B. Specht, "Ven $\mu$ s geometric image quality commissioning" Proc. SPIE 10785, Sensors, Systems, and NextGeneration Satellites XXII, 107850J (25 September 2018); https://doi.org/10.1117/12.2325360

Dechoz, C., et al. "Sentinel 2 global reference image." Image and Signal Processing for Remote Sensing XXI. Vol. 9643. International Society for Optics and Photonics, 2015.

Dedieu, G., Karnieli, A., Hagolle, O., Jeanjean, H., Cabot, F., Ferrier, P., \& Yaniv, Y. (2006). Venus: a joint French Israeli Earth observation mission with high spatial and temporal resolution capabilities. In RAQRS II conference.

Delvit, J. M., Greslou, D., Amberg, V., Dechoz, C., Delussy, F., Lebegue, L., ... \& Bernard, L. (2012). Attitude assessment using Pleiades-HR capabilities. International Archives of the Photogrammetry, Remote Sensing and Spatial Information Sciences, 39(B1), 525-530.

Henri P. Gavin, 2019 , The Levenberg-Marquardt algorithm for nonlinear least squares curve-fitting problems, http://people.duke.edu/ hpgavin/ce281/lm.pdf

De Lussy, F., Greslou, D., \& Colzy, L. G. (2008, January). Process line for geometrical image correction of disruptive microvibrations. In International Society for Photogrammetry and Remote Sensing (pp. 27-35). 
Perrier, R., Arnaud, E., Sturm, P., \& Ortner, M. (2014). Estimation of an observation satellite's attitude using multimodal pushbroom cameras. IEEE transactions on pattern analysis and machine intelligence, 37(5), 987-1000.

A.Rolland, R.Binet, A.Dick, J-L.Raynaud, L.Mongin, G.Dedieu, M.Déjus, "DEM generation from native stereo Ven $\mu$ s acquisitions". COSPAR 2019 Symposium communication. Doi : 10.5281/zenodo.3632935

Roques, S., Brachere, F., Rougé, B., Pausader, M. (2001). Séparation des décalages induits par l'attitude et le relief entre images d'un couple stéréoscopique. In $18^{\circ}$ Colloque sur le traitement du signal et des images, FRA, 2001. GRETSI, Groupe d'Etudes du Traitement du Signal et des Images.

Jeremy Topaz, Francesc Tinto, Olivier Hagolle, "The Venus super-spectral camera," Proc. SPIE 6361, Sensors, Systems, and Next-Generation Satellites X, 63611E (3 October 2006).

Triggs B., McLauchlan P.F., Hartley R.I., Fitzgibbon A.W., 2010, Bundle Adjustment - A Modern Synthesis, https://hal.inria.fr/inria-00523990

Ye, Zhen, et al. "Estimation and analysis of along-track attitude jitter of ZiYuan-3 satellite based on relative residuals of tri-band multispectral imagery." ISPRS Journal of Photogrammetry and Remote Sensing 158 (2019): 188-200. 